\title{
A Near-Optimal Sensor Placement Algorithm to Achieve Complete Coverage/Discrimination in Sensor Networks
}

\author{
Frank Y. S. Lin and P. L. Chiu, Student Member, IEEE
}

\begin{abstract}
In this letter, we develop a robust and scalable algorithm to cope with the sensor placement problem for target location under constraints of the cost limitation and the complete coverage. The problem is NP-complete for arbitrary sensor fields. The grid-based placement scenario is adopted and the sensor placement problem formulated as a combinatorial optimization problem for minimizing the maximum distance error in a sensor field under the constraints. The proposed algorithm is based on the simulated annealing approach. The experimental results reveal that, for small sensor fields, the algorithm can find the optimal sensor placement under the minimum cost limitation. Moreover, it can also find a placement with minimum distance error for large sensor fields under the cost limitation.
\end{abstract}

Index Terms - Sensor placement, target location, mathematical programming, optimization, simulated annealing.

\section{INTRODUCTION}

$\mathbf{I}^{\mathrm{r}}$ $\mathrm{N}$ distributed sensor networks (DSNs), the sensor placement due to cost limitation is currently one of the most important research issues. A sensor network can be deployed in two ways - with random placement or with grid-based placement. When the environment is unknown, random placement is the only choice and sensors may be thrown to any place by aircrafts randomly. The alternative is to deploy sensors on a sensor field to guarantee a particular quality of service, if the properties of the terrain are predetermined. The field is generally divided into grids and sensors are carefully deployed at the grid points. This approach is called grid-based placement. This letter focuses on this method.

Sensor placement strategy depends on the DSN's application. If it is to be used for surveillance, the placing of sensors depends on the coverage. Discrimination must be considered when they are used to solve target location problems.

References [1] and [2] present a resource-bounded optimization framework for sensor resource management under the constraints of sufficient grid coverage of the sensor field. Reference [3] formulates the sensor placement problem in terms of cost minimization under coverage constraints. A

Manuscript received April 15, 2004. The associate editor coordinating the review of this letter and approving it for publication was Prof. Samuel Pierre.

Frank Y. S. Lin is with the Department of Information Management, National Taiwan University, Taipei, Taiwan, R.O.C. (e-mail: yslin@im.ntu. edu.tw).

P. L. Chiu is with the Department of Information Management, National Taiwan University, Taipei, Taiwan, R.O.C. She is also an instructor at the Department of Electronic Engineering, Northern Taiwan Institute of Science and Technology, Taipei, Taiwan, R.O.C. (e-mail: d7725001@im.ntu.edu.tw). Digital Object Identifier 10.1109/LCOMM.2005.01027.

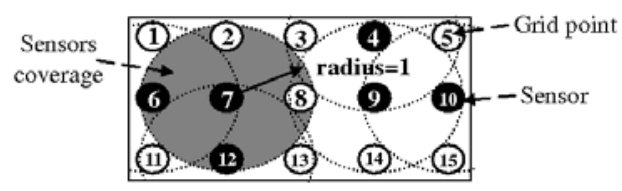

Fig. 1. A complete covered and discriminated sensor field.

framework for identifying codes is applied to determine sensor placement for a unique target location. However, this approach cannot be applied to irregular sensor fields. According to our survey, the sensor placement for target location has not been solved by mathematic optimization methodology.

\section{PROBLEM DESCRIPTION}

A grid-based sensor field can be represented as a collection of two- or three-dimensional grid points [1]. A set of sensors can be deployed on the grid points to monitor the sensor field. In this letter, we consider the detection model of a sensor to be a $0 / 1$ coverage model. The coverage is assumed to be full (1) if the distance between the grid point and the sensor is less than the detection radius of the sensor. Otherwise, the coverage is assumed to be ineffective (0). If any grid point in a sensor field can be detected by at least one sensor, we call the field is completely covered, as shown in Fig. 1. In this context, a target can be detected at any place in the field.

A power vector is defined for each grid point to indicate whether sensors can cover a grid point in a field. As shown in Fig. 1, the power vector of grid point 8 is $(0,0,1,1,0,0)$ corresponding to sensor $4,6,7,9,10$, and 12 . In a completely covered sensor field, when each grid point is identified by a unique power vector, the sensor field is said to be completely discriminated, as shown in Fig. 1. In this case, as soon as a target occurs in a grid of the sensor field, it can be located by the back-end according to the power vector of the grid.

Sometimes, due to some resource limitations, a completely discriminated sensor field cannot be constructed. Consequently, these may lead to wrong determinations, whenever a target occurs at any one of the indistinguishable grid points. Positioning accuracy, therefore, becomes a major consideration in solving the problem. Distance error is one of the most natural criteria to measure positioning accuracy. The distance error of two indistinguishable grid points is defined as the Euclidean distance between them. In this letter, we intend to 
minimize the distance error when complete discrimination is not possible.

\section{MAThematical Model}

The sensor placement problem is formulated herein as a combinatorial optimization problem. The formulation can plan a sensor network that provides either complete or high, discrimination, depending on the cost limitation. Complete discrimination requires that the minimum Hamming distance of the power vectors associated with any pair of grid points be at least one. High discrimination requires that the maximum distance error be minimized. The problem is, therefore, defined as a min-max model.

\section{Given Parameters:}

$A=\{1,2, \ldots, m\}$ : Index set of the sensors' candidate locations.

$B=\{1,2, \ldots, n\}:$ Index set of the locations in the sensor field, $m \leq n$.

$r_{k}$ : Detection radius of the sensor located at $k, k \in A$.

$d_{i j}$ : Euclidean distance between location $i$ and $j, i, j \in B$.

$c_{k}$ : The cost of the sensor allocated at location $k, k \in A$.

$G$ : Total cost limitation.

\section{Decision Variables:}

$y_{k}: 1$, if a sensor is allocated at location $k$ and 0 otherwise, $k \in A$.

$v_{i}=\left(v_{i 1}, v_{i 2}, \ldots, v_{i k}\right):$ The power vector of location $i$, where $v_{i k}$ is 1 if the target at location $i$ can be detected by the sensor at location $k$ and 0 otherwise, where $i \in B, k \in A$.

\section{Objective Function:}

Subject to :

$$
Z_{I P 1}=\min _{v} \max _{(i, j)} \frac{d_{i j}}{1+K \sum_{k=1}^{m}\left(v_{i k}-v_{j k}\right)^{2}}
$$

$$
\begin{aligned}
v_{i k} d_{i k} & \leq y_{k} r_{k} & & , \forall k \in A, i \in B, i \neq k \\
\frac{d_{i k}}{r_{k}} & >y_{k}-v_{i k} & & , \forall k \in A, i \in B, i \neq k \\
v_{i k} & =y_{k} & & , \forall k \in A, i \in B, i=k \\
\sum_{k=1}^{m} c_{k} y_{k} & \leq G & & \\
\sum_{k=1}^{m} v_{i k} & \geq 1 & & , \forall i \in B \\
v_{i k}, y_{k} & =0 \text { or } 1 & & , \forall k \in A, i \in B .
\end{aligned}
$$

( $K$ is an arbitrarily large number.)

When $\sum\left(v_{i k}-v_{j k}\right)^{2}=0$, objective function (IP1) introduces a penalty $d_{i j}, d_{i j} \geq 1$. As $K \rightarrow \infty$ and $\sum\left(v_{i k}-v_{j k}\right)^{2}>$ $0, Z_{I P 1}$ introduces a penalty $d_{i j} /(1+K)$, which approaches zero.

Constraints (1), (2), and (3) require the relationship between sensor detection radius $r_{k}$ and detection distance $d_{i k}$. If a target appears at grid point $i$ and the grid is inside the coverage of sensor $k$, the sensor can detect the target if sensor $k$ is

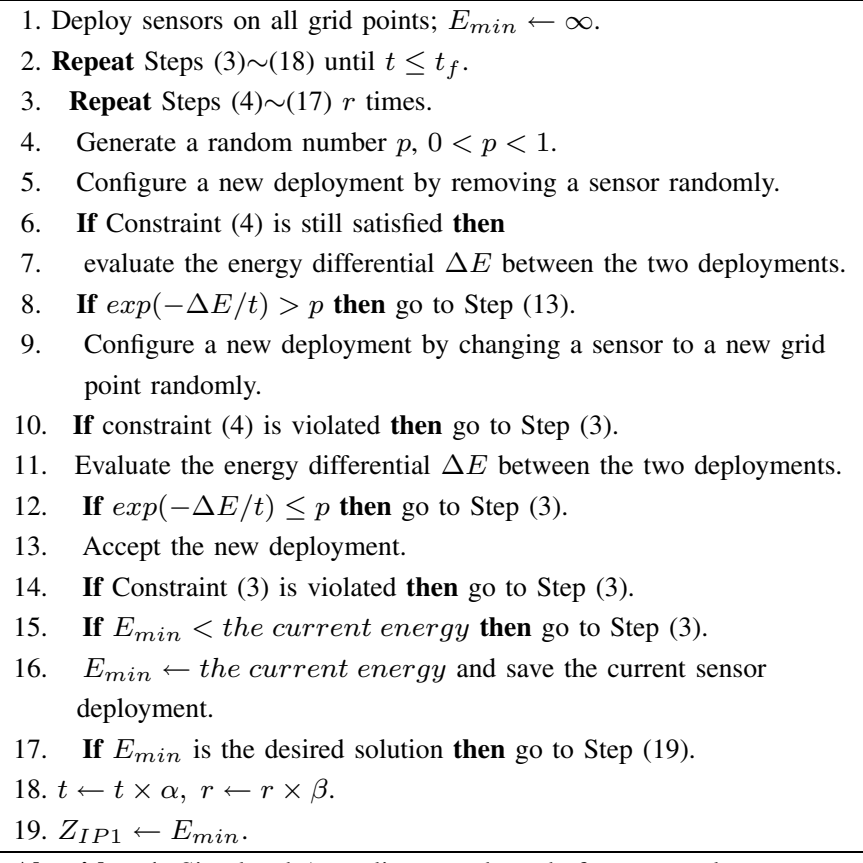

Algorithm 1: Simulated Annealing pseudo code for sensor placement.

available. Constraint (4) requires that the total deployment cost of sensors be limited by cost $G$. Constraint (5) is the complete coverage limitation. Constraint (6) is an integer constraint.

\section{Algorithm}

Simulated annealing (SA) is a highly reliable method for solving hard combinatorial optimization problems. The concept of SA is applied to derive an efficient method for solving the problem approximately.

Here, the cooling schedule of the algorithm is stated briefly. Initially, we assume the sensors are deployed at all grid points. In each loop, an attempt is made to remove one sensor if the cost constraint is not met. Otherwise, an attempt is made to move a sensor to another randomly chosen position. Moreover, the stopping criterion is modified to improve efficiency. Besides frozen temperature, $t_{f}$, is reached, when both complete coverage and discrimination are achieved, such that $Z_{I P 1}=1 /(1+K)$, the procedure will then be stopped. The solution with complete coverage and discrimination may not be optimal. However, the solution is the desired solution to this problem.

Algorithm 1 shows a pseudo code of the algorithm. The energy, $E$, is defined as follows:

$$
E=\max _{(i, j)} \frac{d_{i j}}{1+K \sum_{k=1}^{m}\left(v_{i k}-v_{j k}\right)^{2}} .
$$

\section{Computational Results}

This section presents the computational results. First, the performance of the proposed algorithm is evaluated when small sensor fields are deployed. The purpose of the experiment is to examine whether the algorithm can find the optimal solution under a minimum cost constraint. Then, the performance results in the case of larger sensor fields are presented under various cost constraints. 
TABLE I

COMPARISON BETWEEN EXHAUSTIVE SEARCH AND THE SA ALGORITHM.

\begin{tabular}{|c|c|c|c|c|c|c|c|}
\hline \multirow[b]{2}{*}{ Area } & \multicolumn{2}{|c|}{$\#$ of sensors } & \multirow{2}{*}{$\begin{array}{l}\text { Sensor } \\
\text { density }\end{array}$} & \multirow[b]{2}{*}{ Area } & \multicolumn{2}{|c|}{ \# of sensors } & \multirow{2}{*}{$\begin{array}{l}\text { Sensor } \\
\text { density }\end{array}$} \\
\hline & Opt. & SA & & & Opt. & SA & \\
\hline $3 \times 4$ & 4 & 4 & $44.44 \%$ & $6 \times 4$ & 10 & 10 & $41.67 \%$ \\
\hline $4 \times 3$ & 6 & 6 & $50 \%$ & $6 \times 5$ & 12 & 12 & $40 \%$ \\
\hline $4 \times 4$ & 7 & 7 & $43.75 \%$ & $7 \times 3$ & 9 & 9 & $42.86 \%$ \\
\hline $5 \times 3$ & 6 & 6 & $40 \%$ & $7 \times 4$ & 12 & 12 & $42.86 \%$ \\
\hline $5 \times 4$ & 8 & 8 & $40 \%$ & $8 \times 3$ & 10 & 10 & $41.67 \%$ \\
\hline $5 \times 5$ & 10 & 10 & $40 \%$ & $9 \times 3$ & 11 & 11 & $40.74 \%$ \\
\hline $6 \times 3$ & 8 & 8 & $44.44 \%$ & $10 \times 3$ & 12 & 12 & $40 \%$ \\
\hline
\end{tabular}

Note: Opt. is found by exhaustive search.

The parameters of the cooling schedule are $\alpha=0.75$ and $\beta=1.3$. The initial values of $r$ and $t$ are $5 n$ and 0.1 , respectively; and $n$ is the amount of grids in the sensor field. The frozen temperature, $t_{f}$, is set to $t_{0} / 30 . K$ is 10000 and the cost of sensor, $c_{i}, \forall 1 \leq i \leq n$, is set to one.

As all sensors have the same deployment cost, the cost constraint, Constraint (4), can be expressed as a limit on the number of sensors. This section uses a normalized term, sensor density, in the constraint. Sensor density is defined as follows:

$$
\text { Sensor density }(\%)=\left(\sum_{k=1}^{m} \frac{y_{k}}{n}\right) \times 100 \% \text {. }
$$

\section{A. Experiment I}

Experiment I evaluates the performance of the proposed algorithm for smaller rectangular sensor fields that have no more than 30 grid points. The results are compared with those obtained in an exhaustive search.

First, we find a minimum sensor density for a complete covered and discriminated sensor field. Then, an attempt is made to obtain the same result by using the proposed algorithm under a sensor density constraint.

Table 1 shows the results. In all cases, the proposed algorithm achieves the same deployment of sensor fields with a minimum sensor density. The required sensor density ranges between $40 \%$ and $45 \%$, except when the rectangular sensor field is $4 \times 3$. The exhaustive search for the $10 \times 3$ sensor field exceeds 65 minutes. However, the proposed algorithm finds the solution in 0.1 second.

\section{B. Experiment II}

In this experiment, two larger sensor fields, with $10 \times 10$ and $30 \times 30$ grid points, are considered. The radius of the sensor is one. The values of $Z_{I P 1}$ are determined for various sensor densities. The results obtained using the proposed algorithm is compared with the best solution obtained by the random placement approach. The best solution that has a minimum objective value is found in 1000 arbitrarily generated solutions.

The time to compute 1000 arbitrary solutions for the $30 \times 30$ sensor field with $70 \%$ sensor density is 700 seconds. That for the proposed algorithm is only a couple of minutes. Figs. 2 and 3 show that the required density for the desired solution obtained using the proposed algorithm ranges from $40 \%$ to $45 \%$.

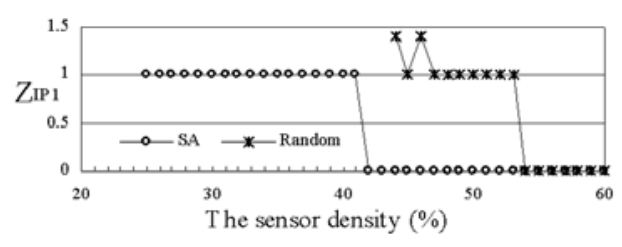

Fig. 2. Results for Exp. II (10 by 10 grid points).

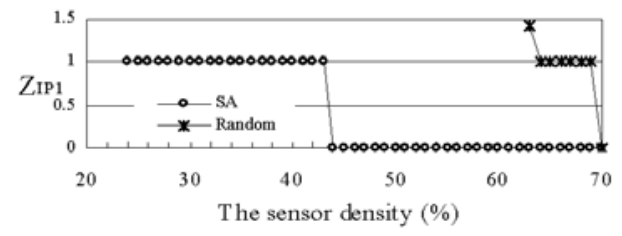

Fig. 3. Results for Exp. II (30 by 30 grid points).

This result is consistent with Table 1 . In contrast, the random placement approach is associated with a relatively high density (54\% and $69 \%$ for Figs. 2 and 3, respectively). The proposed algorithm is, therefore, very effective and scalable.

Figs. 2 and 3 indicate that the placement of sensors by the proposed algorithm has a minimum distance error of one, when the sensor density is insufficient. The random placement approach cannot achieve the same result.

The proposed algorithm can achieve completely covered placement at a very low sensor density. The minimum required sensor densities in Figs. 2 and 3 are only $25 \%$ and 24\%, respectively. The results are very close to the theoretical lower bound. (When the sensor radius is $1^{+}$, a sensor can cover 5 grid points. Hence, the lower bound of the sensor density for complete coverage is 20\%). However, with the random placement approach, the required density for completely covered placement is very high ( $44 \%$ and $63 \%$ for Figs. 2 and 3, respectively). The results show that the probability of finding the feasible solution using the random placement approach is very low when the area of the sensor field increases.

\section{CONClusions}

This letter considers the sensor placement problem for locating targets under cost constraints. We first formulate this problem as a min-max mathematical optimization model where the accuracy of discrimination is the objective. Then, the simulated annealing-based algorithm is developed to solve the optimization problem. The experimental results show that the proposed algorithm can efficiently obtain a high-quality solution. Additionally, the proposed algorithm is very effective, scalable, and robust.

\section{REFERENCES}

[1] S. S. Dhillon, K. Chakrabarty, and S. S. Iyengar, "Sensor placement for grid coverage under imprecise detections," Proc. of the 15th Intl. Conference on Information Fusion, vol. 2, pp. 1581-1587, July 2002.

[2] S. S. Dhillon and K. Chakrabarty, "Sensor placement for effective coverage and surveillance in distributed sensor networks," Proc. of IEEE WCNC 2003, vol. 3, pp. 1609-1614, Mar. 2003.

[3] K. Chakrabarty et al., "Grid coverage for surveillance and target location in distributed sensor networks," IEEE Trans. Computers, vol. 51, pp. 1448-1453, Dec. 2002. 